\title{
The Gödel Completeness Theorem for Uncountable Languages $^{1}$
}

\author{
Julian J. Schlöder \\ Mathematisches Institut \\ Rheinische Friedrich-Wilhelms-Universität Bonn \\ Endenicher Allee 60 \\ D-53113 Bonn, Germany \\ Peter Koepke \\ Mathematisches Institut \\ Rheinische Friedrich-Wilhelms-Universität Bonn \\ Endenicher Allee 60 \\ D-53113 Bonn, Germany
}

Summary. This article is the second in a series of two Mizar articles constituting a formal proof of the Gödel Completeness theorem [15] for uncountably large languages. We follow the proof given in [16]. The present article contains the techniques required to expand a theory such that the expanded theory contains witnesses and is negation faithful. Then the completeness theorem follows immediately.

MML identifier: GOEDCPUC, version: $\underline{7.14 .014 .183 .1153}$

The notation and terminology used here have been introduced in the following papers: [8], [1], [3], [10], [19], [5], [14], [11], [12], [7], [6], [22], [2], [4], [17], [18], [23], [20], [9], [21], and [13].

\footnotetext{
${ }^{1}$ This article is part of the first author's Bachelor thesis under the supervision of the second author.

(C) 2012 University of Białystok CC-BY-SA License ver. 3.0 or late ISSN $1426-2630(\mathrm{p}), 1898-9934(\mathrm{e})$
} 


\section{Formula-Constant Extension}

For simplicity, we use the following convention: $A_{1}$ denotes an alphabet, $P_{1}$ denotes a consistent subset of CQC-WFF $A_{1}, P_{2}$ denotes a subset of CQC-WFF $A_{1}, p, q, r, s$ denote elements of CQC-WFF $A_{1}, A$ denotes a non empty set, $J$ denotes an interpretation of $A_{1}$ and $A, v$ denotes an element of the valuations in $A_{1}$ and $A, n, k$ denote elements of $\mathbb{N}, x$ denotes a bound variable of $A_{1}$, and $A_{2}$ denotes an $A_{1}$-expanding alphabet.

Let us consider $A_{1}$ and let $P_{1}$ be a subset of CQC-WFF $A_{1}$. We say that $P_{1}$ is satisfiable if and only if:

(Def. 1) There exist $A, J, v$ such that $J \models{ }_{v} P_{1}$.

In the sequel $J_{2}$ is an interpretation of $A_{2}$ and $A$ and $J_{1}$ is an interpretation of $A_{1}$ and $A$.

One can prove the following proposition

(1) There exists a set $s$ such that for all $p, x$ holds $\langle s,\langle x, p\rangle\rangle \notin \operatorname{Symb} A_{1}$.

Let us consider $A_{1}$. A set is called a free symbol of $A_{1}$ if:

(Def. 2) For all $p, x$ holds $\langle$ it, $\langle x, p\rangle\rangle \notin \operatorname{Symb} A_{1}$.

Let us consider $A_{1}$. The functor FCEx $A_{1}$ yielding an $A_{1}$-expanding alphabet is defined as follows:

(Def. 3) $\quad$ FCEx $A_{1}=\mathbb{N} \times\left(\operatorname{Symb} A_{1} \cup\left\{\left\langle\right.\right.\right.$ the free symbol of $\left.\left.\left.A_{1},\langle x, p\rangle\right\rangle\right\}\right)$.

Let us consider $A_{1}, p, x$. The example of $p$ and $x$ yielding a bound variable of FCEx $A_{1}$ is defined as follows:

(Def. 4) The example of $p$ and $x=\left\langle 4,\left\langle\right.\right.$ the free symbol of $\left.\left.A_{1},\langle x, p\rangle\right\rangle\right\rangle$.

Let us consider $A_{1}, p, x$. The example formula of $p$ and $x$ yielding an element of CQC-WFF FCEx $A_{1}$ is defined by:

(Def. 5) The example formula of $p$ and $x=\neg \exists_{\text {FCEx } A_{1} \text {-Cast } x}\left(\right.$ FCEx $A_{1}$-Cast $\left.p\right) \vee$ (FCEx $A_{1}$-Cast $p$ ) (FCEx $A_{1}$-Cast $x$, the example of $p$ and $x$ ).

Let us consider $A_{1}$. The example formulae of $A_{1}$ yields a subset of CQC-WFF FCEx $A_{1}$ and is defined as follows:

(Def. 6) The example formulae of $A_{1}=\{$ the example formula of $p$ and $x\}$.

One can prove the following proposition

(2) Let $k$ be an element of $\mathbb{N}$. Suppose $k>0$. Then there exists a $k$-element finite sequence $F$ such that

(i) for every natural number $n$ such that $n \leq k$ and $1 \leq n$ holds $F(n)$ is an alphabet,

(ii) $F(1)=A_{1}$, and

(iii) for every natural number $n$ such that $n<k$ and $1 \leq n$ there exists an alphabet $A_{2}$ such that $F(n)=A_{2}$ and $F(n+1)=$ FCEx $A_{2}$. 
Let us consider $A_{1}$ and let $k$ be a natural number. A $k+1$-element finite sequence is said to be a FCEx-sequence of $A_{1}$ and $k$ if it satisfies the conditions (Def. 7).

(Def. 7)(i) For every natural number $n$ such that $n \leq k+1$ and $1 \leq n$ holds $\operatorname{it}(n)$ is an alphabet,

(ii) it $(1)=A_{1}$, and

(iii) for every natural number $n$ such that $n<k+1$ and $1 \leq n$ there exists an alphabet $A_{2}$ such that $\operatorname{it}(n)=A_{2}$ and $\operatorname{it}(n+1)=$ FCEx $A_{2}$.

The following propositions are true:

(3) For every natural number $k$ and for every FCEx-sequence $S$ of $A_{1}$ and $k$ holds $S(k+1)$ is an alphabet.

(4) For every natural number $k$ and for every FCEx-sequence $S$ of $A_{1}$ and $k$ holds $S(k+1)$ is an $A_{1}$-expanding alphabet.

Let us consider $A_{1}$ and let $k$ be a natural number. The $k$-th FCEx of $A_{1}$ yielding an $A_{1}$-expanding alphabet is defined as follows:

(Def. 8) The $k$-th FCEx of $A_{1}=$ the FCEx-sequence of $A_{1}$ and $k(k+1)$.

Let us consider $A_{1}, P_{1}$. A function is called an EF-sequence of $A_{1}$ and $P_{1}$ if it satisfies the conditions (Def. 9).

(Def. 9)(i) dom it $=\mathbb{N}$,

(ii) $\quad$ it $(0)=P_{1}$, and

(iii) for every natural number $n$ holds $\operatorname{it}(n+1)=\operatorname{it}(n) \cup$ the example formulae of the $n$-th FCEx of $A_{1}$.

Next we state two propositions:

(5) For every natural number $k$ holds FCEx (the $k$-th FCEx of $\left.A_{1}\right)=$ the $(k+1)$-th FCEx of $A_{1}$.

(6) For all $k, n$ such that $n \leq k$ holds the $n$-th FCEx of $A_{1} \subseteq$ the $k$-th FCEx of $A_{1}$.

Let us consider $A_{1}, P_{1}$ and let $k$ be a natural number. The $k$-th EF of $A_{1}$ and $P_{1}$ yields a subset of CQC-WFF (the $k$-th FCEx of $A_{1}$ ) and is defined as follows:

(Def. 10) The $k$-th EF of $A_{1}$ and $P_{1}=$ the EF-sequence of $A_{1}$ and $P_{1}(k)$.

One can prove the following propositions:

(7) For all $r, s, x$ holds $A_{2}$-Cast $(r \vee s)=A_{2}$-Cast $r \vee A_{2}$-Cast $s$ and $A_{2}$-Cast $\exists_{x} r=\exists_{A_{2}-\text { Cast } x}\left(A_{2}\right.$-Cast $\left.r\right)$.

(8) For all $p, q, A, J, v$ holds $J \models_{v} p$ or $J \models_{v} q$ iff $J \models_{v} p \vee q$.

(9) $\quad P_{1} \cup$ the example formulae of $A_{1}$ is a consistent subset of CQC-WFF FCEx $A_{1}$. 


\section{The Completeness Theorem}

We now state four propositions:

(10) There exists an $A_{1}$-expanding alphabet $A_{2}$ and there exists a consistent subset $P_{2}$ of CQC-WFF $A_{2}$ such that $P_{1} \subseteq P_{2}$ and $P_{2}$ has examples.

(11) $P_{1} \cup\{p\}$ is consistent or $P_{1} \cup\{\neg p\}$ is consistent.

(12) Let $P_{2}$ be a consistent subset of CQC-WFF $A_{1}$. Then there exists a consistent subset $T_{1}$ of CQC-WFF $A_{1}$ such that $T_{1}$ is negation faithful and $P_{2} \subseteq T_{1}$.

(13) For every consistent subset $T_{1}$ of CQC-WFF $A_{1}$ such that $P_{1} \subseteq T_{1}$ and $P_{1}$ has examples holds $T_{1}$ has examples.

Let us consider $A_{1}$. One can check that every subset of CQC-WFF $A_{1}$ which is consistent is also satisfiable.

We now state the proposition

$(14)^{2} \quad$ If $P_{2}=p$, then $P_{2} \vdash p$.

\section{REFERENCES}

[1] Grzegorz Bancerek. Cardinal numbers. Formalized Mathematics, 1(2):377-382, 1990.

[2] Grzegorz Bancerek. The fundamental properties of natural numbers. Formalized Mathematics, 1(1):41-46, 1990.

[3] Grzegorz Bancerek. König's theorem. Formalized Mathematics, 1(3):589-593, 1990.

[4] Grzegorz Bancerek. The ordinal numbers. Formalized Mathematics, 1(1):91-96, 1990.

[5] Grzegorz Bancerek and Krzysztof Hryniewiecki. Segments of natural numbers and finite sequences. Formalized Mathematics, 1(1):107-114, 1990.

[6] Patrick Braselmann and Peter Koepke. Equivalences of inconsistency and Henkin models. Formalized Mathematics, 13(1):45-48, 2005.

[7] Patrick Braselmann and Peter Koepke. Gödel's completeness theorem. Formalized Mathematics, 13(1):49-53, 2005.

[8] Patrick Braselmann and Peter Koepke. A sequent calculus for first-order logic. Formalized Mathematics, 13(1):33-39, 2005.

[9] Patrick Braselmann and Peter Koepke. Substitution in first-order formulas. Part II. The construction of first-order formulas. Formalized Mathematics, 13(1):27-32, 2005.

[10] Czesław Byliński. A classical first order language. Formalized Mathematics, 1(4):669-676, 1990.

[11] Czesław Byliński. Functions and their basic properties. Formalized Mathematics, 1(1):5565, 1990.

[12] Czesław Byliński. Functions from a set to a set. Formalized Mathematics, 1(1):153-164, 1990.

[13] Czesław Byliński. Some basic properties of sets. Formalized Mathematics, 1(1):47-53, 1990.

[14] Agata Darmochwał. Finite sets. Formalized Mathematics, 1(1):165-167, 1990.

[15] Kurt Gödel. Die Vollständigkeit der Axiome des logischen Funktionenkalküls. Monatshefte für Mathematik und Physik 37, 1930.

[16] W. Thomas H.-D. Ebbinghaus, J. Flum. Einführung in die Mathematische Logik. Springer-Verlag, Berlin Heidelberg, 2007.

[17] Piotr Rudnicki and Andrzej Trybulec. A first order language. Formalized Mathematics, 1(2):303-311, 1990.

\footnotetext{
${ }^{2}$ Completeness Theorem.
} 
[18] Julian J. Schlöder and Peter Koepke. Transition of consistency and satisfiability under language extensions. Formalized Mathematics, 20(3):193-197, 2012, doi: 10.2478/v10037012-0022-0.

[19] Andrzej Trybulec. Domains and their Cartesian products. Formalized Mathematics, 1(1):115-122, 1990.

[20] Zinaida Trybulec. Properties of subsets. Formalized Mathematics, 1(1):67-71, 1990.

[21] Edmund Woronowicz. Interpretation and satisfiability in the first order logic. Formalized Mathematics, 1(4):739-743, 1990.

[22] Edmund Woronowicz. Many argument relations. Formalized Mathematics, 1(4):733-737, 1990.

[23] Edmund Woronowicz. Relations and their basic properties. Formalized Mathematics, $1(\mathbf{1}): 73-83,1990$.

Received May 7, 2012 